\section{Oral diagnosis and treatment planning: part 2. Dental caries and assessment of risk}

\author{
K. Yip ${ }^{1}$ and R. Smales ${ }^{2}$
}

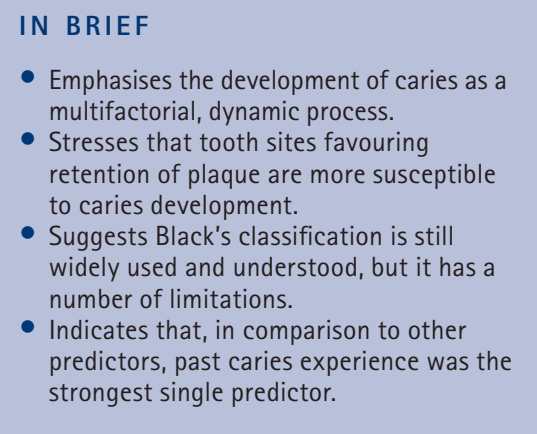

Dental caries or tooth decay may be defined as a dynamic process causing progressive destruction of hard tooth substance (enamel, dentine and cementum) involving demineralisation of the inorganic portion of the tooth, and dissolution of the organic portion. The onset and progression of carious lesions involves multiple host, micro-organism and substrate factors interacting in a continuous flux. The diagnosis of initial lesions remains a challenge for practitioners and, despite numerous studies, the assessment of future caries risk is still based largely on a patient's past caries experience. If caries is allowed to progress then pulpitis will occur, which may result in subsequent pulpal necrosis and lead to a local periapical and perhaps a systemic infection.

\section{AETIOLOGY OF DENTAL CARIES}

The development of dental caries is a multifactorial, dynamic process. Mineralised tooth tissues are not inert, as the minerals they contain are in a state of constant demineralisation/remineralisation flux

\begin{tabular}{|c|c|}
\hline \multicolumn{2}{|c|}{$\begin{array}{l}\text { ORAL DIAGNOSIS } \\
\text { AND TREATMENT PLANNING* }\end{array}$} \\
\hline Part 1. & $\begin{array}{l}\text { Introduction to oral diagnosis } \\
\text { and treatment planning }\end{array}$ \\
\hline Part 2. & Dental caries and assessment of risk \\
\hline Part 3. & $\begin{array}{l}\text { Periodontal disease and assessment } \\
\text { of risk }\end{array}$ \\
\hline Part 4. & $\begin{array}{l}\text { Non-carious tooth surface loss } \\
\text { and assessment of risk }\end{array}$ \\
\hline Part 5. & $\begin{array}{l}\text { Preventive and treatment } \\
\text { planning for dental caries }\end{array}$ \\
\hline Part 6. & $\begin{array}{l}\text { Preventive and treatment } \\
\text { planning for periodontal disease }\end{array}$ \\
\hline Part 7. & $\begin{array}{l}\text { Treatment planning for } \\
\text { missing teeth }\end{array}$ \\
\hline Part 8. & $\begin{array}{l}\text { Reviews and maintenance } \\
\text { of restorations }\end{array}$ \\
\hline \multicolumn{2}{|c|}{$\begin{array}{l}\text { *This series represents chapters 1, 7, 8, 9, 14, 15, } 16 \text { and } 19 \text { from } \\
\text { the } B D J \text { book A Clinical Guide to Oral Diagnosis and Treatment } \\
\text { Planning, edited by Roger Smales and Kevin Yip. All other } \\
\text { chapters are published in the complete clinical guide available } \\
\text { from the } B D J \text { Books online shop. }\end{array}$} \\
\hline
\end{tabular}

${ }^{1}$ Adjunct Professor, School of Dentistry, Charles Sturt University, Orange, New South Wales 2800, Australia; ${ }^{2} \mathrm{~V}$ isiting Research Fellow, School of Dentistry, Faculty of Health Sciences, The University of Adelaide, Adelaide, South Australia 5005, Australia

${ }^{*}$ Correspondence to: Roger J. Smales

Email: roger.smales@adelaide.edu.au

Accepted 7 June 2012

DOI: 10.1038/sj.bdj.2012.615

${ }^{\circledR}$ British Dental Journal 2012; 213: 59-66 with the oral environment. Caries results when the net demineralising flux prevails over the net remineralising flux. In the classical model, plaque micro-organisms, fermentable carbohydrate substrate, a susceptible tooth surface, and time are involved in the initiation and progression of dental caries (Fig. 1).

\section{Micro-organisms}

These are present in an amorphous mass of biofilm known as dental plaque, which adheres to the tooth surface via the acquired dental pellicle (a collection of adherent proteins on the tooth surface, derived from the saliva). In addition to the micro-organisms, plaque also contains food debris, minerals and a gelatinous polysaccharide matrix (glucans and fructans) synthesised by some species of bacteria present in the plaque (Fig. 2). The quantity of plaque increases with time, and this increase in bulk is accompanied by a change in its quality, becoming more Gram-negative and anaerobic. It is the fermentation of saccharide by the anaerobic/facultative anaerobic microorganisms that produces the acids (mainly lactic), which demineralise the inorganic tooth substance. The main bacteria implicated in this process are Streptococcus mutans, S. sanguis, S. salivarius, Actinomyces viscosus, A. naeslundii and Lactobacillus species.

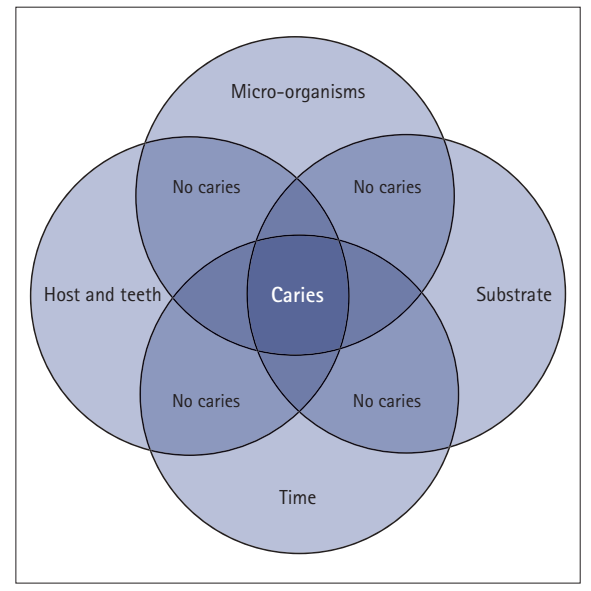

Fig. 1 The Venn diagram emphasises that all four factors must be present and acting together for caries to occur and to progress (Oxford University Press: with permission)

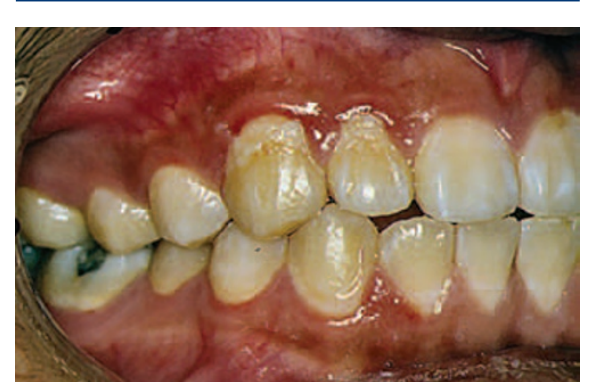

Fig. 2 Thick plaque and food material have accumulated, associated with acute gingivitis adjacent to the maxillary canine and lateral incisor following inadequate oral hygiene

\section{Substrate}

The carbohydrates fermented by cariogenic bacteria diffuse into the dental plaque from the oral environment. Not all sugars 
are equally cariogenic, high molecular weight complex carbohydrates such as starch, which are not completely digested in the mouth, have a low cariogenicity. Low molecular weight simple carbohydrates such as glucose, fructose and lactose, which easily diffuse into plaque and are transported into bacterial cells, have a high cariogenicity. Since sucrose is the most common form of refined sugar consumed, it is the most important sugar in the development of caries.

\section{HOST FACTORS}

\section{Tooth morphology}

Tooth sites that favour the retention of plaque are more susceptible to the development of caries. These include:

- Deep narrow enamel pits and fissures

- Smooth surfaces adjacent to the approximal contact areas

- Smooth surfaces adjacent to the gingival margins

- Exposed root surfaces

- Rough deficient margins of restorations

- Surfaces adjacent to prostheses (removable dentures and appliances, fixed prostheses, Figs 3 and 4).

\section{Saliva and crevicular fluid}

These contain minerals, especially calcium and phosphate, and buffers that neutralise the acids produced by bacteria and enhance the remineralisation of demineralised lesions. In addition, they contain IgA secretory antibody and lysozyme whose exact roles are still unclear, but may be significant as anti-cariogenic factors. Saliva also has the physical effect of washing away debris from tooth surfaces. The important contribution of saliva in caries prevention may be seen in those persons with uncontrolled hyposalivation (decreased salivary flow) in whom rampant caries often occurs.

Apart from extensive caries, severe hyposalivation with associated xerostomia also causes many other upper digestive tract problems that significantly decrease the quality of life of affected persons. Such persons require an aggressive preventive and therapeutic approach to relieve their discomfort and pain, and to prevent further oral and other health problems. Apart from the identification and possible removal of the causes of hyposalivation, professional efforts should enhance the salivary flow and buffering capacity, tooth surface integrity, and tooth surface protection. Patients should be given detailed printed home care instructions, including the use when necessary of commercial nonirritating dentifrices that do not contain sodium lauryl sulphate, and saliva substitutes that do not contain alcohol, acids, glycerine or sugars. Suitable widely available products include those from the Biotène Dry Mouth (Laclede Inc.) range.

\section{Fluoride}

Fluoride ions in the saliva, dental plaque and pellicle, and tooth substance enhance the remineralisation of carious lesions provided that adequate amounts of calcium and phosphate ions are available. At high concentrations, fluoride ions also inhibit the carbohydrate metabolism of cariogenic bacteria. It is thought that this reducing environment is the major anti-cariogenic effect of fluoride rather than incorporation into developing tooth tissue per se. Thus, current concepts of fluoride use favour frequent topical applications at low doses, rather than the use of infrequent systemic high doses, to reduce the potential for tooth demineralisation.

\section{Time}

The fermentation of sugars by cario-genic bacteria, the increase in plaque bulk and the demineralisation of tooth tissue all require a minimum length of time for the net demineralising flux to become greater than the net remineralising flux.

\section{CLASSIFICATION OF CARIOUS LESIONS}

\section{By tooth surface site and by size}

Early in the 20th century G.V. Black classified carious lesions according to their location on the tooth crown as follows:

- Class I: Caries affecting the pits and fissures in the occlusal surfaces of posterior teeth, the facial and lingual surfaces of molar teeth, and the palatal surfaces of maxillary anterior teeth

- Class II: Caries affecting the proximal surfaces of posterior teeth

- Class III: Caries affecting the proximal surfaces of anterior teeth

- Class IV: Caries affecting the proximal

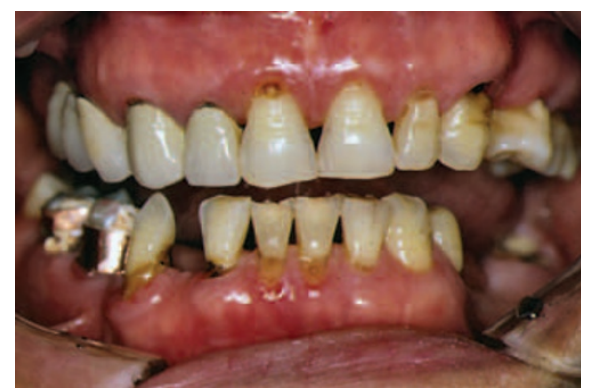

Fig. 3 Retention of plaque around the cervical margins of the maxillary anterior and posterior ceramo-metal crowns has resulted in secondary caries

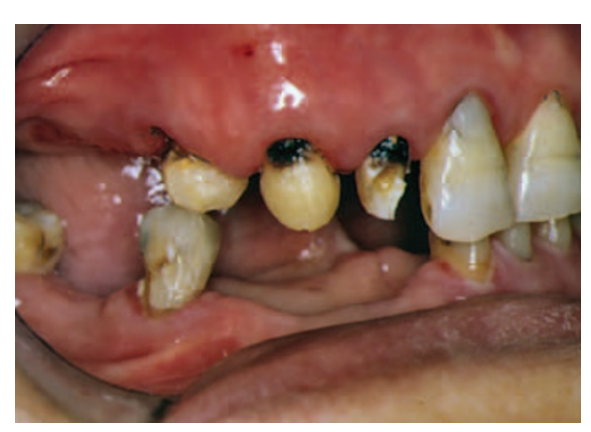

Fig. 4 Removal of the crowns revealed advanced root caries of the restored teeth

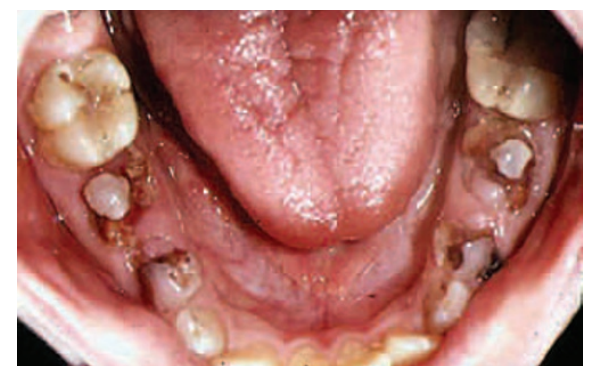

Fig. 5 Acute caries involving many teeth has resulted in rampant carious lesions in this child. Pulp polyps are present in the grossly carious primary second molars

surfaces of anterior teeth, and involving the incisal angle

- Class V: Caries affecting the cervical one-third of facial and lingual surfaces, but not involving pits and fissures

- Class VI: Caries affecting the incisal edges of anterior teeth and the cusp tips of posterior teeth.

Black's classification is still widely used and understood, but it has a number of limitations, notably that it does not include secondary caries (caries occurring around the margins of existing restorations), or root surface (cemental) caries, both of which are important reasons for operative treatment. Kidd and Smith ${ }^{1}$ have suggested a simpler, broader classification of carious lesions according to susceptible sites, as follows: 


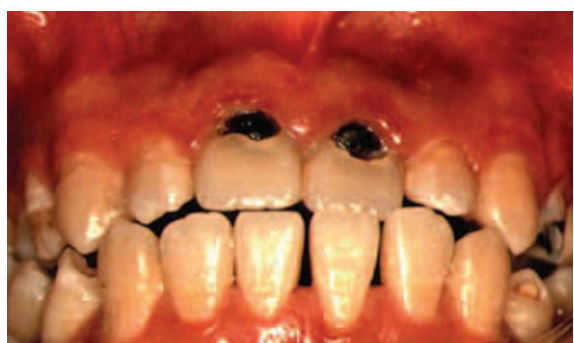

Fig. 6 Black, exposed cervical dentine in the maxillary central incisors indicates a slowing of the carious lesions

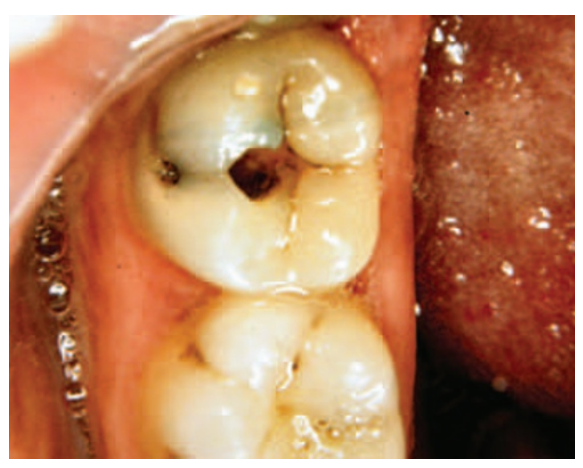

Fig. 7 Primary caries that originated in the occlusal and buccal enamel pits and fissures

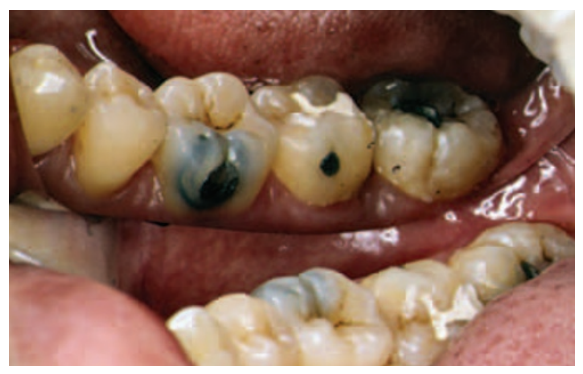

Fig. 8 Secondary caries associated with an amalgam restoration placed to restore a buccal pit lesion. The amalgam in the second molar is sound

- Caries of the anatomical enamel pits and fissures (Black's Class I)

- Caries of the approximal enamel smooth surfaces (Black's Class II, III and IV)

- Caries of the cervical enamel margins at the gingival margin, or of the root surfaces where gingival recession is present (Black's Class V and root surface caries)

- Caries around the margins of existing restorations (secondary caries/ recurrent caries).

However, because modern operative dentistry is far more conservative compared to that practiced previously, another carious lesion classification has been proposed by Mount and Hume, ${ }^{2}$ based on the tooth crown/root surface site, as well as on

Table 1 Classification of carious lesions by cavity site and size

\begin{tabular}{l|l|l|l|l|l} 
Site & No cavity (0) & Minimal (1) & Moderate (2) & Enlarged (3) & Extensive (4) \\
\hline Pit or fissure (1) & 1.0 & 1.1 & 1.2 & 1.3 & 1.4 \\
\hline Contact area (2) & 2.0 & 2.1 & 2.2 & 2.3 & 2.4 \\
\hline Cervical region (3) & 3.0 & 3.1 & 3.2 & 3.3 & 3.4 \\
\hline
\end{tabular}

cavity presence and size which is linked to treatments (Table 1). Minimal cavities show minimal surface cavitation and dentine involvement that cannot be treated by remineralisation alone.

- Size 0: Initial stages of demineralisation - no restoration required

- Size 1: Minimal surface cavitation with involvement of dentine, just beyond remineralisation - restoration is required

- Size 2: Moderate involvement of dentine - enamel is sound and well supported by dentine after cavity preparation and the tooth is sufficiently strong enough to support the restoration under normal occlusal load

- Size 3: Enlarged cavity after caries removal - the remaining tooth structure is weakened and further enlargement of the cavity is needed to protect the weak tooth structure

- Size 4: Extensive caries or bulk loss of tooth structure has occurred.

\section{By tooth surface integrity}

The tooth surface affected by the carious lesion may be non-cavitated (intact) or cavitated. The former condition can be managed by non-operative means, while the latter situation requires either a restoration or the removal of adjacent tooth substance to allow the effective mechanical removal of plaque retained within the lesion.

\section{By progression rate}

Caries (the decay process) can be defined as being active (acute or chronic) or inactive:

- Acute: the process undergoes a rapid progressive clinical course, with early pulp involvement overwhelming the pulpal defences, and with severe pain. The exposed dentine is usually pale yellow/light brown and soft. 'Rampant caries' occurs when many teeth and normally caries-immune tooth surfaces are involved (Fig. 5)

- Chronic: the process progresses slowly, or becomes inactive (arrested) when the dental biofilm can be removed, and the amount of sclerotic and reactionary or tertiary dentine formed is often adequate to protect the pulp, with perhaps an occasional dull ache or pain present. The exposed dentine is usually dark brown/black and leathery (Fig. 6).

Caries progression rates tend to be faster in persons with large amounts of retained dental plaque, low salivary flow rates and buffering capacities, and low tooth fluoride content.

\section{By initial or subsequent activity}

Caries can be defined as primary or secondary:

- Primary caries attacks a previously intact tooth surface (Fig. 7)

- Secondary caries (recurrent caries) develops either at the margins or adjacent to the margins of a restoration. Its origin and progress is the same as for primary caries. It may be difficult to distinguish advanced secondary caries from residual caries (that which is not removed during cavity preparation). This remaining caries may continue to progress when the restoration fails to seal the lesion from the oral environment (Fig. 8).

\section{PREVALENCE AND INCIDENCE OF DENTAL CARIES}

Both the prevalence (occurrence at one point in time in a population) and the incidence (rate over time in a population) of dental caries increase with advances in civilisation and the increased availability of refined sugars. However, after a long period of continued decrease, recent trends 
in several developed countries now show an increase in caries prevalence, especially in children and young adults. The incidence of dental caries is closely related to dietary habits and oral hygiene practices, which are related to socio-economic conditions. Thus, in part, different populations exhibit different caries incidences. In general the following pattern applies in developed countries:

- Caries is more common in economically, socially, medically and mentally compromised persons

- Females have a slightly higher caries rate than males

- Teenagers have the highest incidence of pit and fissure caries

- Persons in their early twenties have the highest incidence of interproximal lesions

- Persons over 60 years old have the highest incidence of root caries

- The highest incidence of hidden caries in the occlusal surfaces of permanent molars occurs in countries having widespread use of fluorides

- Heredity appears to play a relatively minor role in the susceptibility to caries

- Dental caries appears to develop with approximate bilateral symmetry. Berman and Slack ${ }^{3}$ carried out annual dental inspections of 353 11-year-old schoolgirls. After three years they found caries in $74 \%$ of mandibular second molars, $62 \%$ of mandibular first molars, and 63\% of maxillary first and second molars. All of these teeth had pit and fissure 'stagnation areas'. The mandibular incisors were found to be relatively immune to caries, due to the protective action of saliva

- Geographic variables include fluorides which, when present in water supplies in a proportion of around 1 part per million, can reduce the caries rate by approximately 50\%. Soil types could be important since certain trace elements such as molybdenum also inhibit the development of caries. An alkaline soil also appears to be associated with a low incidence of caries.

\section{DIAGNOSIS OF THE PRESENCE AND EXTENT OF CARIOUS LESIONS}

There is considerable disagreement among dentists in their diagnoses of caries and decisions to intervene. and in

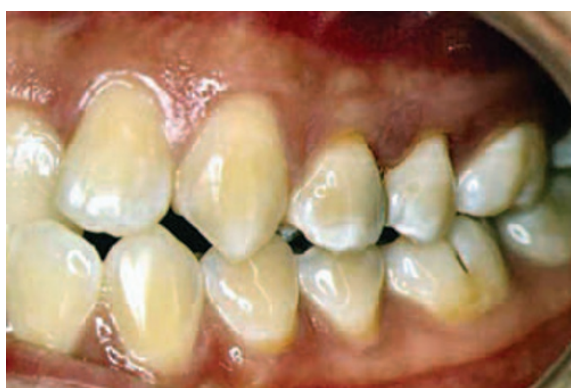

Fig. 9 Initial primary enamel caries visible as opaque cervical 'white spots' in the molars. Inital cervical white spot lesions also are present in Fig. 2

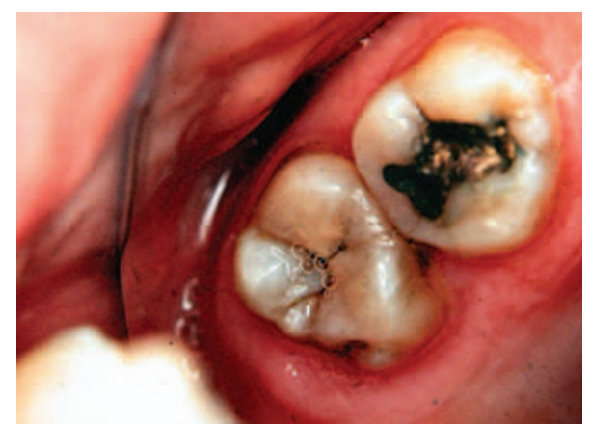

Fig. 10 The grey occlusal opacity in the maxillary third molar is evidence of extensive carious involvement of the underlying dentine
Table 2 Simplified international caries detection and assessment system (ICDAS)

Lesion progression $\quad$ Clinical description

\begin{tabular}{l|l}
\hline Code 0 & Sound enamel surface, when air-dried for five seconds. \\
\hline Code 1 & First visual change in enamel, only observed when surface is dried. \\
\hline Code 2 & Distinct visual change in enamel, also observed when surface is wet. \\
\hline Code 3 & Localised enamel breakdown, with or without underlying dentine involvement shadow. \\
\hline Code 4 & Distinct cavity with visible dentine, which is soft to gentle blunt probing. \\
\hline $\begin{array}{l}\text { For root surfaces code } 0=\text { sound surface (exclude abrasion/erosion); code } 1 \text { = distinct visual colour } \\
\text { change when surface is dried but no cavitation; code } 2=\text { cavitation is also present (loss of anatomical } \\
\text { contour } \geq 0.5 \text { mm). }\end{array}$ \\
\multicolumn{2}{l}{ Sourced from Criteria Manual (ICDAS-II), Baltimore workshop 2005 }
\end{tabular}

their selection of treatments. A systematic review ${ }^{4}$ of six commonly used methods to identify carious lesions found that the strength of evidence to allow general estimates of their sensitivity and specificity was poor for most applications. Caries diagnosis requires clean, dry teeth, good lighting and good visual access.

\section{Visual and tactile examinations}

These are the only clinical methods that can be used to distinguish between active and inactive carious lesions.

\section{Visual}

Initial demineralisation appears as an opacity or dull white spot lesion in the enamel, which is more easily detected when the tooth surfaces are dried for approximately five seconds (Fig. 9). The initial lesion also may appear as a light brown discoloration due to the uptake of surface stains. Heavy coffee and tea drinking and smoking may confound the diagnosis of initial lesions. Progression of enamel caries leads to a chalky appearance with some minor local loss of surface integrity resulting in surface roughness. More advanced lesions often appear as a darkening or bluish grey lesion within the tooth, due to the carious involvement of the underlying dentine beneath intact or largely intact enamel (Fig. 10). This is particularly obvious beneath the occlusal surfaces of permanent molar teeth (hidden caries or 'fluoride bombs'), where enamel strengthening from fluorides has resulted in apparently intact occlusal fissures, rather than open occlusal cavities. The same darkening also may be seen beneath the intact occlusal enamel surface of a proximal marginal ridge, due to an underlying proximal carious lesion extending into the dentine. Further caries progression results in cavitation in the opaque or discoloured enamel, exposing the underlying carious dentine.

\section{Tactile}

Blunt dental explorers or probes may be used to determine gently, the consistency of a suspected carious lesion. Care must be exercised to avoid damage caused by firm pressure with a sharp explorer, in particular to non-cavitated enamel and root surfaces.

An International Caries Detection and Assessment System (ICDAS) has been proposed for assessing the progression 


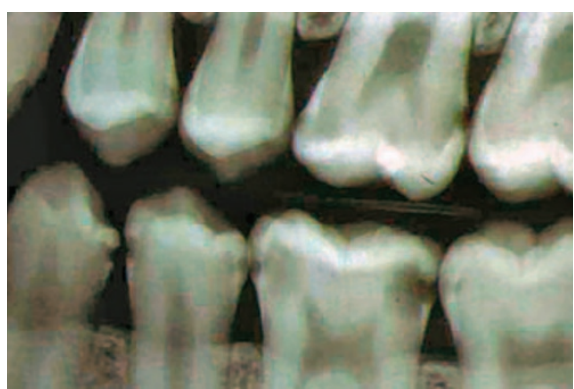

Fig. 11 Active, rampant caries of multiple proximal tooth surfaces. The larger carious lesions in dentine appear to have extended to the pulp horns in the mandibular first molar

of primary and secondary carious lesions in coronal and root surfaces. The clinical descriptions reflect the underlying histopathology of carious lesion progression. The lesions are classified, with the assistance of a blunt dental explorer, as either active or inactive. Inactive remineralised and arrested carious lesions usually appear hard and shiny or leathery, and either whitish, brownish or black. Examiner reproducibility and accuracy for the diagnosis of the presence or not of occlusal caries has generally been rated as good. A simplified general description is shown in Table 2. Localised enamel breakdown (Code 3) cannot be treated by remineralisation alone.

\section{Separators}

Mechanical separators may be used to gain immediate direct vision and access to check enamel surface integrity and for the treatment of small proximal lesions with localised enamel surface breakdown. Orthodontic elastic separators may be placed for several days to achieve similar, but slower tooth separation.

\section{Transillumination}

Fibre-optic lights are usually employed to detect proximal surface carious lesions, which appear as dark areas. Lesions are seen most easily in anterior and premolar teeth where the contact areas are narrower bucco-lingually than in molars. The method has a rather low sensitivity.

\section{Laser and blue light fluorescence examination}

An electronic caries detector, the DIAGNOdent (KaVo), is used widely for the diagnosis of initial occlusal carious lesions. However, it should be used as an adjunct to traditional diagnostic methods,

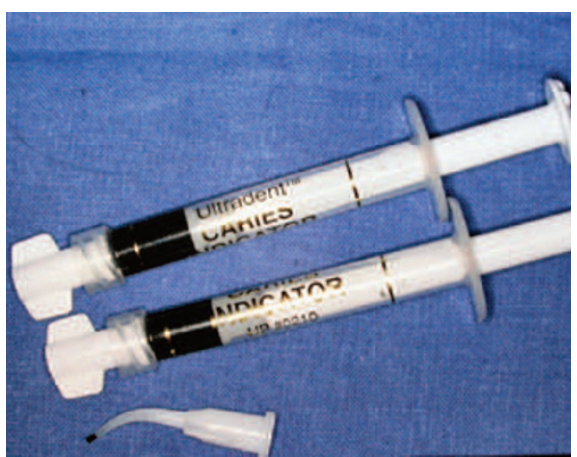

Fig. 12 A caries-detector dye intended to stain only bacteria-infected carious dentine

as its use alone may lead to many falsepositive results for occlusal pit and fissure caries in low caries risk populations in particular. False-positive results may also occur from calculus, organic material, resin composites, fluorescent prophylaxis pastes, and areas of enamel hypoplasia. The DIAGNOdent pen (KaVo) also may be used for the additional detection of proximal lesions.

Recommended cut-off limits for the DIAGNOdent device are: 0-13 (no caries), 14-20 (enamel caries, with preventive care advised), 21-30 (dentine caries, with preventive or operative care advised depending on the caries- risk assessment), >30 (operative care advised). A much lower value of 5-10, for optimal sensitivity and specificity, has been advised for the diagnosis of root surface caries. The device may also be used to monitor changes over time at different tooth sites.

Several commercial products such as Spectra Caries Detection Aid (Air Techniques) that emit LED blue light have now been marketed for the diagnosis of initial occlusal pit and fissure caries according to colour-coded and numerical responses. As with other fluorescencebased diagnostic systems, false-positive results may occur.

Under the high-intensity visible blue light from a curing wand, white spot and pre-visible white spot lesions appear as dark areas against the yellow fluorescence of sound tooth enamel when viewed through an orange protective shield.

\section{Electrical conductance examination}

Sensitivity and specificity findings of approximately 90\% have been obtained from assessing occlusal pits and fissures for carious lesions when employing the CarieScan PRO (CarieScan). The results from using AC impedance spectroscopy are displayed as colour-coded and numerical responses.

\section{Radiographic examination}

This is important for the detection of small interproximal carious lesions in enamel, and for larger occlusal carious lesions extending into dentine. Carious lesions appear as a radiolucency (darkening or shadowing) on radiographs. Proximal concavities in cervical root surfaces may be confused with proximal root caries. Radiographs may not reveal the presence of many small carious lesions, as the histologic extent of the actual lesions is usually much larger than their radiographic appearances, especially in active progressive lesions (Fig. 11). Therefore, in patients with a high risk of dental caries where the lesions cannot be controlled over a six-month period and show penetration into dentine on radiographs, restorations should be placed.

\section{Chemical examination}

Nonspecific dyes have been developed that will stain demineralised and infected carious lesions to varying degrees. The dyes can be taken up by demineralised porous enamel and have been used to examine the occlusal pits and fissures of posterior teeth in children in particular, where there is less stainable organic material present to confound the diagnosis of caries. However, there are often no or very low levels of micro-organisms present, even when the enamel demineralisation extends into dentine below non-cavitated enamel.

Other, so-called, caries-detector dyes may be used to differentiate between infected and affected dentinal lesions (Fig. 12). However, the dyes stain altered collagen and not the micro-organisms present in the lesions. The dyes also stain lessmineralised, normal dentine over the pulp horns and at the enamel-dentinal junction, leading to inadvertent pulp exposures and the over-preparation of cavities when attempting to remove all of the pale dye-stained non-infected dentine from cavity preparations.

Therefore, the use of dyes for caries detection will result in many false positives for incipient pit and fissure lesions because of the presence of retained organic debris in the fissures, and will also result in the 
over-preparation of cavities when carious dentine is present because of the staining of largely non-infected demineralised or affected dentine.

\section{DIFFERENTIAL DIAGNOSIS}

The two conditions most often confused with the initial appearances of dental caries are those of dental fluorosis and other enamel hypoplasias. Dental fluorosis is a developmental condition and, therefore, has a symmetrical distribution on similar homologous teeth. In mild cases, fine glossy white horizontal lines follow the enamel surface perichymata. Cusp tips, incisal edges and marginal ridges may show 'snow caps'. The tooth surfaces are affected according to the duration and severity of exposure to excessive fluorides. More severe cases show enamel surface pitting and staining.

Other (non-fluoride related) enamel hypoplasias may involve either individual teeth, or also arise from a systemic cause. Glossy white roundish, usually well defined, opacities may be found on one or a few teeth. Most are found in the incisal two-thirds region of the crown. More severe cases may have enamel surface defects and pronounced yellow-brown staining.

\section{ASSESSMENT OF RISK TO DENTAL CARIES}

Epidemiological and clinical studies have shown that the risk of developing dental caries differs between individuals. In common with many other diseases, some individuals are more at risk of developing caries than others. Moreover, even for the same individual there may be times when the caries risk changes. Therefore, an assessment of the current caries risk of patients under your care is essential so that appropriate treatment plans can be proposed. Patients assessed as being at high risk would be targeted for intensive preventive treatments and frequent recalls. Conversely, those patients assessed as being at low risk to caries and other oral diseases would not require such intensive management.

Despite considerable research, most of the individual tests used to assess future caries risk have limited predictive validity. Past caries experience remains both the most reliable and most practical method for use in dental practice to assess future caries risk in patients. However, it must be expected that some patients who are at high caries risk will not be identified. The assessment of caries risk involves an element of prediction and, as with all predictions, is not always entirely correct. But, an attempt at prediction and the provision of appropriate treatment is better than providing the same 'blanket' prevention and treatment to everyone. In one multi-practice study, general practitioners identified, with little variability, an average of approximately 5\% (range 0-18\%) of their patients as being at high risk to either dental caries or periodontal disease. Moderate-risk and low-risk patients were less readily identified, with much larger variability between practitioners.

\section{Past caries experience}

The past caries experience of the patient is probably the most frequently used factor in the assessment of caries risk. In comparison to other predictors, past caries experience was the strongest single predictor.

The sensitivity of prediction varies, but based on previous caries experience, usually approximately $60 \%$ of individuals at high risk of further caries are correctly identified. However, this does mean that approximately $40 \%$ are incorrectly identified as being at high risk. Untreated active carious lesions (cavitated and/or non-cavitated) in permanent and primary teeth are among the strongest predictors for practitioners classifying patients to higher risk groups. Carious lesions involving proximal tooth surfaces and recently-erupted tooth surfaces were the most significant for such classifications. Active lesions affecting the mandibular incisors and/or perhaps the buccal surfaces of the maxillary molars, where salivary flow rates are usually rapid, also indicate a high risk situation. Two or more new active lesions in one year usually indicate a person to be at high risk.

In 6-year-old children, the extent of caries in the primary dentition (decayed, missing and filled teeth: DMFT), appears to be a better predictor for caries at the age of 7-13 years, than is the extent of caries in the first permanent molars. In adults, where there may be a considerable proportion of tooth surfaces filled, the DMFT score may be a less reliable predictor of coronal caries than in young persons. This is because a high DMFT score is historic only, and indicates a previous high caries activity. The caries risk of an individual may alter because of changes in diet, fluoride use and general health, but this alteration would not necessarily be indicated in the DMFT, as the D, M and F components cannot be reduced.

There is also an association between the past coronal caries experience and the risk of developing root-surface caries, and an association between root-surface caries scores and the future root-surface caries experience.

\section{Microbiological tests}

The use of microbiological tests as a means of identifying caries risk is based upon caries being caused by a microbial infection. The numbers and types of cariogenic micro-organisms present in saliva are used, since there is a correlation between these micro-organisms and those found in plaque.

The main microbiological tests involve counts of lactobacilli and mutans streptococci. Due to their positive numerical association with human caries and the linkage of this association to carbohydrate consumption, such tests may serve potentially not only as a caries risk predictor, but also as an indicator of carbohydrate consumption, another caries risk factor. However, the use of lactobacilli counts as a screening test for caries risk has been found to be of limited value. Similarly, the predictive power of mutans streptococci in saliva has not proven better than that of the past caries experience. These factors, combined with the time and costs required to conduct such microbiological tests mean that, despite their simplification with chairside results now possible, such tests will probably not feature much in general dental practice.

\section{Saliva}

The protective role of saliva is well known, and is based on its volume and buffering capacity combined with its antimicrobial properties. The two factors most commonly measured to determine caries risk are the stimulated salivary flow rate and its buffering capacity. Severe reduction in the flow rate of saliva is known to predispose teeth to caries. Hyposalivation can occur in patients who take medications that reduce salivary flow, who have 


\begin{tabular}{|c|c|c|}
\hline \multicolumn{3}{|c|}{$\begin{array}{l}\text { The types of food and drink you consume can influence tooth decay and acidic tooth erosion. Following the } \\
\text { examination of your teeth, it would be helpful to look at your diet. Advice can then be given to you on the } \\
\text { types of food and drink that are least harmful to your teeth. }\end{array}$} \\
\hline \multicolumn{3}{|c|}{$\begin{array}{l}\text { Instructions } \\
\text { Over a period of four full days (which must include one weekend), you should write down the details of } \\
\text { all foods, drinks, sweets or candies, chewing gums, medicines, etc, that you place in your mouth. You must } \\
\text { write down the date and time, name and quantity of the food or drink, etc, as accurately as possible. If } \\
\text { you do not know the name of the food or drink then give a brief description. }\end{array}$} \\
\hline Name: & Day: & Date: \\
\hline Time & Name of food, drink, etc & Quantity \\
\hline 10:00 am & Toast with banana & 2 slices, 1 banana \\
\hline 11:00 am & Vita-Soy drink & 1 bottle \\
\hline 11:00 am & Egg tart & 2 pieces \\
\hline $11: 55 \mathrm{am}$ & Coca-Cola drink & 1 can \\
\hline
\end{tabular}

received irradiation to the head and neck, and who have other medical conditions that adversely affect saliva production.

Although a negative correlation between caries risk and the buffering capacity of saliva has been found in some studies, the test sensitivity is so low that it cannot be used solely in screening for high caries risk. Thus, apart from identifying those persons who, due to their medical condition, have a reduced salivary flow and are potentially at high risk to caries, other salivary tests are of probably of less importance in dental practice.

\section{Diet}

The relationship between fermentable carbohydrates, especially sucrose, and dental caries has been clearly established. However, because of often incorrect recordings, and the often short period of recording, the self-reported sucrose intake obtained from a dietary analysis may have limited value. The objective of the analysis is to educate the patient, to eliminate sticky between-meal sugary snacks and drinks, and to advise on healthier foods and sugar substitutes, including noncariogenic sweeteners such as aspartame, saccharin, sorbitol and xylitol. A similar estimate of acidic food and drink ingestion also may be included for education of the patient, together with appropriate advice on preventing tooth erosion, and tooth surface damage from incorrect brushing after erosive episodes. A high ingestion of refined carbohydrates may be associated with 'nursing-bottle caries', poor socioeconomic conditions, and some occupations, medical conditions, medicaments and illicit drugs, and difficulties in chewing. An assessment of the dietary analysis as part of the Traffic Light-Matrix Model is described in chapter 13 of the $B D J$ clinical guide associated with this $B D J$ series. ${ }^{5}$ An abbreviated example of a dietary analysis form is shown in Table 3.

\section{Oral hygiene}

The relationship between caries risk and either the amount of plaque on the teeth or the frequency of self-reported oral hygiene measures is poor. However, poor oral hygiene may be useful in adults and the elderly for identifying an increased risk of root caries, and possibly useful in children for identifying an increased risk of pit and fissure caries.

\section{Social factors}

In industrialised countries, persons having a poor education and low socio-economic status tend to have more carious lesions than people with a high socio-economic status. Therefore, it may be useful to consider the social background of patients when assessing the caries risk.

\section{Combination of different factors}

It would be expected that by combining some of the various tests, this could improve the reliability of predicting high risk to caries. An example would be the past history of caries experience combined with a microbial test. In some studies this has been found to improve the test reliability, but not substantially. In one study, researchers analysed data from 30 clinical, microbiological, sociodemographic and dental health behavioural factors and found that, with only one exception, information based on clinical examinations provided the only statistically highly significant predictor. And, microbiological predictors were not useful.

Several of these, and other, biological risk factors for dental caries are regarded as important considerations when planning preventive and restorative treatment options for individual patients. A systematic risk assessment method employing the Traffic Light-Matrix (TL-M) management model for dental caries as part of the treatment planning process is discussed in chapter 13 of $B D J$ clinical guide. ${ }^{5}$

\section{FURTHER READING}

Bader J D, Shugars D A. Understanding dentists' restorative treatment decisions. J Public Health Dent 1992; 52: 102-110.

Bader J D, Shugars D A, Kennedy J E, Hayden W J, Baker S. A pilot study of riskbased prevention in private practice. $J \mathrm{Am}$ Dent Assoc 2003; 134: 1195-1202.

International Caries Detection and Assessment System (ICDAS) Coordinating Committee. Criteria manual: International Caries Detection and Assessment System (ICDAS II). Workshop held in Baltimore, Maryland, March 12-14 2005.

Disney J A, Graves R C, Stamm J W, Bohannan H M, Abernathy J R, Zack D D. The University of North Carolina Caries Risk Assessment study: further developments in caries risk prediction. Community Dent Oral Epidemiol 1992; 20: 64-75.

Fejerskov 0, Kidd E. Dental caries: the disease and its clinical management. 2nd ed. Oxford: Blackwell Munksgaard, 2008.

Jablonski-Momeni A, Stachniss V, Ricketts D N, Heinzel-Gutenbrunner M, Pieper K. Reproducibility and accuracy of the ICDAS-II for detection of occlusal caries in vitro. Caries Res 2008; 42: 79-87.

Kidd E A M. Essentials of dental caries: the disease and its management. 3rd ed. Oxford: Oxford University Press, 2005.

Lussi A, Megert B, Longbottom C, Reich E, Francescut P. Clinical performance of a laser fluorescence device for detection of occlusal caries lesions. Eur J Oral Sci 2001; 109: 14-19.

McComb D. Caries-detector dyes - how accurate and useful are they? J Can Dent Assoc 2000; 66: 195-198. 
Mount G J. Tooth preparation for restoration with plastic materials. In Mount G J, Hume W R (eds) Preservation and restoration of tooth structure. pp 121-153. St Louis: Mosby, 1998.

Rytömaa I, Jarvinen V, Jarvinen J. Variation in caries recording and restorative treatment plan among university teachers. Community Dent Oral Epidemiol 1979; 7: 335-339.

Saemundsson S R, Slade G D, Spencer
A J, Davies M J. The basis for clinicians' caries risk grouping in children. Pediatr Dent 1997; 19: 331-338.

Smales R J, Yip K H K. Prevention and control of tooth erosion. In Yip K H K, Smales $\mathrm{R} J$, Kaidonis J A. Tooth erosion: prevention and treatment. pp 36-46. New Delhi: Jaypee Brothers Medical Publishers, 2006.

Yip K H K, Stevenson A G, Beeley J A. The use of two caries detector dyes in cavity preparation. Br Dent J 1994; 176: 417-421.
1. Kidd E A M, Smith B G N. Pickard's manual of operative dentistry. 7th ed. Oxford: Oxford University Press, 1996.

2. Mount G J, Hume W R. A new classification for dentistry. Quintessence Int 1997; 28: 301-303.

3. Berman D S, Slack G L. Susceptibility of tooth surfaces to carious attack. A longitudinal study. BrDent J 1973; 134: 135-139.

4. Bader J D, Shugars D A, Bonito A J. A systematic review of the performance of methods for identifying carious lesions. J Public Health Dent 2002; 62: 201-213.

5. Ngo H, Sim C. Traffic light - matrix management model for dental caries. In Yip K H K, Smales R J (eds) A clinical guide to oral diagnosis and treatment planning. pp 106-117. London: BDA Books, 2012. 